Taka Oguisso

Enfermeira e Advogada. Professora Titular do Departamento de Orientação Profissional da Escola de Enfermagem da Universidade de São Paulo, SP.

takaoguisso@usp.br

Genival Fernandes de Freitas

Enfermeiro e Advogado. Professor Doutor do Departamento de Orientação Profissional da Escola de Enfermagem da Universidade de São Paulo, SP. genivalf@usp.br

Submissão: $18 / 09 / 2006$

Aprovação: 12/12/2006

\section{Enfermeiros prescrevendo medicamentos: possibilidades e perspectivas}

\author{
Nurses prescribing medications: possibilities and perspectives \\ Enfermeros prescribiendo medicaciones: posibilidades y perspectivas
}

ATUALIZAÇÃO

\section{RESUMO}

Esforços para assegurar a implementação da prescrição de medicamentos por enfermeiros têm sido um desafio em diversas partes do mundo. Este estudo teve por objetivo descrever como essa prescrição vem sendo realizada em alguns países, e refletir sobre a legislação do exercício profissional de enfermagem no Brasil acerca dessa questão. Dados do Conselho Internacional de Enfermeiras (CIE) e Programas de Saúde Pública do Ministério da Saúde demonstram a necessidade de atuação política mais forte dos enfermeiros e dos seus órgãos representativos, a fim de criar uma legislação que defina melhor sua abrangência e atribuições no que tange à prescrição de medicamentos.

Descritores: Prescrição de medicamentos; Legislação de enfermagem; Assistência à saúde.

\section{ABSTRACT}

Efforts to ensure the implementation of nurses prescribing medications have been a challenge in different parts of the world. This paper aimed at describing how this issue has been accomplished in some countries and reflecting about nursing professional legislation in Brazil in relation to this subject. Data from the International Council of Nurses (ICN) and the Ministry of Health Programs on Public Health show the need for a stronger political action by nurses and their institutional representations in order to create a legislation to better define their scope and functions in relation to prescription of medicines.

Descriptors: Prescription, drugs; Nursing legislation; Delivery of healthcare.

\section{RESUMEN}

Esfuerzos para garantizar la implementación de la prescripción de medicinas por enfermeros han sido un desafío en diversas partes del mundo. Este estudio pretende describir como esta prescripción viene siendo realizada en algunos países y reflexionar sobre la legislación del ejercicio profesional de enfermería en Brasil sobre este tema. Datos del Consejo Internacional de Enfermeras (CIE) y Programas de Salud Pública del Ministerio de Salud, demuestran la necesidad de una actuación política más fuerte de los enfermeros y de sus órganos representativos a fin de crear una legislación que pueda definir mejor el ámbito y funciones de los enfermeros con relación a la prescripción de medicinas.

Descriptores: Prescripción de medicamentos; Legislación de enfermería; Prestación de atención a la salud.

Oguisso T, Freitas GF. Enfermeiros prescrevendo medicamentos: possibilidades e perspectivas. Rev Bras Enferm 2007 mar-abr; 60(2):141-4.

\section{INTRODUÇÃO}

As últimas duas décadas têm testemunhado mudanças significativas no papel e nas funções de enfermeiros em muitos países. 0 trabalho de enfermagem tornou-se mais técnico e mais especializado e o enfermeiro passou a ter maior destaque como membro da equipe multidisciplinar, com seu próprio corpo de conhecimentos para a prestação de cuidados ao cliente. Tais fatos vêm ocorrendo numa época de contenção de custos para a saúde e, ao mesmo tempo, aumento da demanda para novos e dispendiosos tratamentos, o que tem feito governos e profissionais de saúde olharem com atenção redobrada a manutenção da eficácia na administração de recursos humanos e materiais nessa área.

Enfermeiros vêm desenvolvendo seu papel de forma inovadora, ora expandindo ora estendendo suas funções, e a prescrição de medicamentos pode ser vista como uma dessas inovações da profissão de enfermagem, que vem sendo implementada na prática dos enfermeiros em muitos países, desde 0 início da década de 1990 , conforme indica a literatura existente ${ }^{(1)}$. O Conselho Internacional de Enfermeiras (CIE) tem se manifestado acerca dessa matéria de modo criterioso e prudente, dado seu impacto em nível 
mundial. $\mathrm{O}$ CIE publicou documento sobre o assunto, e estabeleceu uma rede de comunicação para que profissionais que já vinham se dedicando a atividades de práticas avançadas pudessem discutir situações e problemas comuns. Com isso, o referido Conselho procurou também definir o nivel de qualificação que deveria ter o profissional para assumir essa prática ${ }^{(1)}$.

Este estudo teve por objetivo descrever como a prescrição de medicamentos vem sendo realizada, em alguns países, por enfermeiros, $\mathrm{e}$ discorrer sobre a legislação do exercício profissional de enfermagem, no Brasil, acerca dessa temática.

De acordo com entendimento do $\mathrm{CIE}$, para que o enfermeiro possa exercer práticas avançadas de enfermagem, inclusive, prescrição de medicamentos, deveria ter como mínimo de formação o grau de mestre em enfermagem, mestrado profissionalizante ou alguma forma de pós-graduação em práticas avançadas ou especializadas de enfermagem. Seria, pois, um profissional com conhecimento especializado e habilidade para decisões complexas, além de competência clínica para a prática de atividades expandidas, cujas características estariam conformadas pelo contexto institucional onde esteja habilitado a exercer a atividade profissional(1).

A prática avançada de enfermagem implica também em um estudo sobre o âmbito dessa prática e de padrões relacionados com a atividade. Um aspecto fundamental no âmbito das práticas avançadas é a competência para prescrever medicamentos, proporcionando ao enfermeiro o apoio para desenvolver sua autonomia profissional e o exercício independente. É fato que 0 interesse pela prescrição de medicamentos por enfermeiros vem crescendo, em muitos países, para atenderà necessidade de melhor provisão de cuidados a pacientes, em especial, os que se encontram em comunidades afastadas ou em zona rural; ao uso mais eficaz de tempo e recursos; à necessidade de enfermeiros melhor usarem sua capacidade ou legitimar um trabalho que já estão executando; ao melhor relacionamento entre profissionais de saúde e à redistribuição de trabalho entre profissionais capacitados e melhor utilização do tempo de trabalho do médico.

De acordo com levantamento feito pelo $\mathrm{CIE}$, foram identificados quatro modelos de enfermeiros prescritores de medicamentos: o independente, autônomo ou substitutivo; o dependente, colaborador, semi-autônomo, complementar ou suplementar; o grupo protocolo e a prescrição alterando horário e dosagem ${ }^{(1)}$.

O profissional de saúde, responsável pela prescrição, assume toda a responsabilidade pela avaliação do cliente, usualmente fazendo um diagnóstico diferencial dentro de uma série de possibilidades sugeridas pelos sinais e sintomas e indica a medicação e tratamento adequados, efetuando a prescrição. Essa categoria de prescritor, na maioria dos países, é limitada a médicos, dentistas e veterinários, mas também enfermeiros em vários países têm esse direito. Tais enfermeiros, em geral, prescrevem em formulários específicos (e nesse caso, um número limitado e definido de medicamentos) ou em formulários comuns como ocorre em muitos estados dos Estados Unidos.

O prescritor dependente é aquele que pode prescrever em colaboração com o prescritor independente, geralmente médico, mas sem necessidade de supervisão direta. O prescritor dependente pode prescrever em formulário comum ou especial, consultando, ou não, o médico. O prescritor dependente não assume a responsabilidade pelo diagnóstico ou exame de avaliação do cliente. Esse tipo de prescrição é muito útil nas consultas subseqüentes do cliente, após ele já ter passado por um médico na primeira consulta. Mas, o enfermeiro não deve se limitar a repetir a medicação, pois deve ter competência para alterar alguns aspectos da prescrição, como dose ou freqüência. Nesse tipo de prescrição, o médico continua responsável, em última instância.

O grupo protocolo é aquele que segue uma instrução escrita específica para administração de determinados medicamentos em uma determinada situação clínica. Essa instrução pode ser elaborada dentro de qualquer instituição, pública ou privada, e aprovada pelo dirigente local. É aplicado para grupos de clientes ou usuários previamente identificados. Esse modelo é amplamente usado no Reino Unido, Austrália e Nova Zelândia, além do Brasil. O grupo protocolo não deve ser visto como prescrição independente, porque apenas permite que o enfermeiro prescreva medicamentos dentro dos termos de um determinado protocolo. O uso do grupo protocolo pode ser o caminho para que enfermeiros possam futuramente prescrever mais, de forma independente. No Reino Unido, os grupos protocolo têm sido usados para que outros profissionais, como fisioterapeutas, psicólogos, optometristas e farmacêuticos, além dos enfermeiros também prescrevam em situações de necessidade ou emergência, embora exista também preocupação com aspectos legais em caso de erros ou falhas.

O quarto modelo é o da alteração de horário e dosagem da prescrição do cliente. O protocolo é por cliente e não por grupo e admite que enfermeiros alterem o horário e a dose de medicamentos específicos. É modelo comumente usado por enfermeiros atuando com pacientes psiquiátricos, diabéticos e de cuidados paliativos. Nota-se que esse tipo também não é caso de prescrição independente, mas administração de medicação sob a autoridade e responsabilidade do médico.

\section{A EXPERIÊNCIA DA PRESCRIÇÃO DE MEDICAMENTOS POR ENFERMEIROS EM ALGUNS PAÍSES}

Já vem sendo largamente utilizada a prescrição de medicamentos por enfermeiros, conforme levantamento realizado pelo Conselho Internacional de Enfermeiras (CIE). Entre esses países foram identificados a Suécia, Austrália, Canadá, Estados Unidos, Reino Unido e Nova Zelândia, como os primeiros a implantarem essa experiência, seguidos da África do Sul, Botsuana, Irlanda e Quênia ${ }^{(1)}$.

Os primeiros países, que implantaram a prescrição de medicamentos por enfermeiros, tinham em comum uma forte liderança e uma organização de enfermagem em nivel nacional bem articulada e com experientes e ativos lobistas que obtiveram a aprovação de leis que favoreceram seus projetos e propostas, além de um sistema educacional na enfermagem que deu a capacitação, confiança e competência para assumir o direito de prescrever. Em todos esses países havia um sistema de saúde e de enfermagem comunitária bem estabelecida, com práticas e funções avançadas para enfermeiros. Nesse caso, a prescrição de medicamentos por enfermeiros constituía uma atraente opção para promover a assistência à saúde com recursos existentes e contenção de custos.

$\mathrm{Na}$ Suécia, é exigida uma capacitação específica para o enfermeiro prescrever duzentos e trinta medicamentos, cuja duração é de dez semanas para especialistas e vinte semanas para não especialistas. A prescrição de medicamentos é feita, por enfermeiros, para idosos e pacientes em cuidados primários. 0 enfermeiro não realiza a prescrição inicial ou para crianças. Alguns médicos suecos argumentam que há implicações advindas da prescrição de medicamentos por enfermeiros, alegando que diagnósticos simplistas poderiam prejudicar os clientes. No entanto, órgãos do governo fizeram avaliação positiva, comprovando que houve melhora do nivel da assistência, da comunicação e do acesso a clientes, a partir da assunção dessa prática profissional pelo profissional enfermeiro(1).

Em relação àAustrália, cada província é autônoma para legislar em matéria de saúde e educação. Em algumas províncias, o enfermeiro prescreve medicamentos, desde a primeira prescrição, sendo dele exigida a formação em nível de mestrado e curso especial de terapia com drogas. Requer-se dos enfermeiros que estejam registrados na província onde atuam profissionalmente para executar a prescrição de drogas. Naquele país, existem protocolos especíicos e listagem de medicamentos que podem ser prescritos, porém muitos desses protocolos limitam-se àárea rural. Há posicionamentos, por parte de algumas entidades médicas, que consideram que a prescrição de medicamentos por enfermeiros tem contribuído para a melhoria dos serviços para clientes.

No Reino Unido, desde 1986, há cursos específicos e intensivos para enfermeiros de saúde pública, com estágios supervisionados, para prescreverem medicamentos. Há também uma lista extensa de medicamentos que podem ser prescritos por enfermeiros, incluindo antibióticos e outros de uso tópico ou oral, destinados a pacientes crônicos com asma, diabetes, 
problemas cardíacos e mentais. O Departamento de Saúde do Reino Unido ratificou a posição de que enfermeiros podem prescrever medicamentos de forma independente e estendida, com base em formulários para esse fim. A atuação dos enfermeiros em relação à prescrição de medicamentos possibilitou melhora da satisfação dos clientes e acesso mais fácil ao atendimento.

Nos Estados Unidos da América do Norte (EUA), cada estado da Federação é autônomo para legislar em matéria de seu interesse, inclusive saúde e educação. Enfermeiros de práticas avançadas podem prescrever medicamentos em setores de nível primário (atendimento ambulatorial) ou secundário de atenção à saúde (atendimento hospitalar). Para tanto, é exigido grau de mestre ou curso especial com estudos sobre diagnósticos e tratamentos de doenças. Enfermeiros anestesistas, obstetrizes e enfermeiros clínicos especialistas também podem prescrever medicamentos. O nivel de autonomia é variável, mas todo enfermeiro de práticas avançadas pode prescrever tudo, exceto medicamentos controlados. Muitos pacientes manifestam melhora no atendimento. Enfermeiros americanos prescrevem menos medicamentos e com menor freqüência que os médicos e estes têm, em conjunto com farmacêuticos, se oposto a esse tipo de prática por enfermeiros.

$\mathrm{Na}$ África do Sul, somente enfermeiros de saúde pública são autorizados pelo Conselho de Enfermagem a prescreverem medicamentos, após um curso especial e obtenção de licença do Conselho de Farmácia. Podem ser prescritos vários esquemas de medicamentos, desde adrenalina, anticoagulantes, corticosteróides, anti-histamínicos a antibióticos. Embora não tenham sido documentados pelos enfermeiros sul-africanos os possiveis benefícios com essa prática, há referências de que houve melhora do serviço de atendimento à saúde, especialmente na área rural. Nesse país, o Conselho Nacional de Saúde autoriza quem pode prescrever medicamentos. Há, entretanto, resistência de grupos médicos à prescrição de medicamentos por enfermeiros, sob a alegação de que pode haver aumento de custo por causa de diagnósticos mal feitos pelos enfermeiros.

O Conselho de Medicina da Nova Zelândia admitiu que outros profissionais da área, como dentistas e obstetrizes, também prescrevem medicamentos, e os enfermeiros de geriatria e de pediatria foram incluídos nessa categoria. Mas, foi-lhes exigida capacitação específica em cuidados paliativos, respiratórios, diabetes, saúde mental, problemas ocupacionais e os relacionados à reprodução humana. Admite-se que o enfermeiro prescreva para clientes idosos, terminais e doenças crônicas relacionadas às especialidades autorizadas. Há vários programas com listas de medicamentos que podem ser prescritos por enfermeiros. Atualmente, o Governo da Nova Zelândia estuda a possibilidade de aumentar o âmbito da prescrição de medicamentos pelos enfermeiros.

A legislação brasileira do Exercício Profissional de Enfermagem (Lei n. ${ }^{\circ}$ 7.498, de 25 de Junho de 1986) prevê a prescrição de medicamentos por enfermeiros, como integrantes da equipe de saúde, quando previamente estabelecidos em programas de saúde coletiva e em rotina aprovada por instituição de saúde. O Conselho Federal de Enfermagem (COFEN) tem buscado normatizar essa ação sobre o direito do enfermeiro de prescrever certos medicamentos, dentro dos parâmetros instituídos na Lei do Exercício Profissional de Enfermagem, Lei n. ${ }^{0} 7.498$, de 25 de Junho de 1986 (2).

\section{A PRESCRIÇÃO DE MEDICAMENTOS POR ENFERMEIROS NO BRASIL}

O COFEN baixou a Resolução N. ${ }^{\circ} 271$, em 12 de Julho de 2002, que regulamenta ações do enfermeiro na consulta, prescrição de medicamentos e requisição de exames ${ }^{(3)}$. Segundo esse documento, o enfermeiro tem autonomia na escolha dos medicamentos e respectiva posologia, respondendo integralmente pelos atos praticados. Assim, a prescrição de medicamentos é uma ação de enfermagem, quando praticadas pelo enfermeiro, como integrante da equipe de saúde. No entanto, os limites legais para a prática desta ação são os Programas de Saúde Pública e rotinas que tenham sido aprovadas em instituições de saúde, pública ou privada.
Faz-se mister que os currículos dos cursos de graduação de enfermagem contemplem o preparo técnico do futuro enfermeiro para realização das ações que envolvem a consulta de enfermagem, a prescrição de medicamentos e a requisição de exames.

Para orientar o enfermeiro quanto à segurança na prescrição de medicamentos, o COFEN baixou a Resolução n. ${ }^{0} 195$, de 18 de fevereiro de 1997, segundo a qual o enfermeiro pode solicitar exames de rotina e complementares ${ }^{(4)}$. Essa Resolução se pautou na própria Lei do Exercício Profissional $n^{0} 7.498 / 86$ e no seu Decreto regulamentador n. ${ }^{\circ}$ 94.406/87(5). Essa resolução encontra-se respaldada nos seguintes programas do Ministério da Saúde: Doenças Sexualmente Transmissiveis/AIDS da Coordenadoria de Assistência à Saúde; Viva Mulher; Assistência Integral; e Saúde da Mulher e da Criança; Controle de Doenças Transmissíveis, dentre outros. Encontra respaldo também nos Manuais de Normas Técnicas publicados pelo mesmo Ministério, tais como: Capacitação de enfermeiros em Saúde Pública para Sistema Único de Saúde - Controle das Doenças Transmissíveis; Pré-natal de baixo risco (1986); Capacitação do instrutor/ supervisor enfermeiro na área de controle da hanseníase (1988); Procedimento para atividade e controle da tuberculose (1989); Normas Técnicas e Procedimentos para utilização dos esquemas de poliquimioterapia no tratamento da hanseníase (1990); Guia de controle de hanseníase (1994); e, Normas de atenção à saúde integral do adolescente, de 1995.

As ações defendidas pelo COFEN para a consulta, solicitação de exames de rotina e complementares e a prescrição de medicamentos são objeto de entendimentos controvertidos, chegando às instâncias judiciárias, onde se indaga: seriam as atribuições privativas de médicos ou poderiam ser compartilhadas com outros profissionais da área da saúde?

Nessa ótica, observa-se que a Lei Estadual n. ${ }^{0} 10.241$, de 17 de Junho de 1999, defende o direito do cliente de ser informado de forma clara, compreensiva e acessível sobre bens e serviços de saúde, inclusive, a assistência de enfermagem prestada e, obviamente, os medicamentos utilizados no tratamento ${ }^{(6)}$.

Indiscutivelmente, uma das atribuições da equipe de enfermagem tem sido a administração de medicamentos, envolvendo a requisição do medicamento à farmácia, utilizando-se a prescrição médica para esse procedimento; também, cabe aos profissionais de enfermagem a manipulação e o preparo do medicamento e a infusão do mesmo no cliente. Todas essas ações, envolvem aspectos legais e éticos de impacto sobre a prática profissional(7).

Aadministração de medicamentos prescritos é um papel fundamental à maioria das equipes de enfermagem. Não é somente uma tarefa mecânica a ser executada em complacência rígida com a prescrição médica. Dessa maneira, a administração de medicamentos exige que o profissional de enfermagem detenha conhecimentos técnico-científicos, além de discernimento para o exercício de juízo profissional ${ }^{(8)}$.

Ademais, a administração de medicamentos prescritos pelo médico pela equipe de enfermagem é fato na grande maioria das instituições de saúde, privadas e públicas. No entanto, a prescrição de medicamentos por enfermeiro não é uma prática homogênea para a maioria dos estabelecimentos de saúde, principalmente os privados. O profissional enfermeiro prescreve medicamentos nos programas de saúde pública e em instituições de saúde, quando há protocolo de rotinas preconizados em determinados estabelecimentos de saúde.

Será que é chegado o momento do enfermeiro participar da prescrição terapêtica de medicamentos? Em que casos, esse profissional teria capacitação para tal ação? A capacitação advém dos programas de especialização desenvolvidos pelas sociedades de especialistas e dos programas de pós-graduação. Portanto, a prescrição de medicamentos por enfermeiros não busca suprir a insuficiência numérica de médicos no atendimento às necessidades da população, mas reconhecer que o profissional enfermeiro tem capacidade e competência para a realização dessa atividade de forma segura, garantindo isenção de risco à clientela assistida. 
Ao se propor essa discussão e reflexão acerca da prescrição de medicamentos por enfermeiros, convém ressaltar que o processo de construção social da identidade profissional da enfermagem especializada ainda não se consolidou, ou seja, as instituições formadoras, construtoras sociais do campo profissional da enfermagem, ainda não se definiram pela figura de um só perfil - generalista ou especialista -e, por isso, esta construção ainda encontra-se inacabada ${ }^{(9)}$.

Sendo assim, a prescrição de medicamentos por enfermeiros tem provocado alguns questionamentos acerca da autonomia do enfermeiro para implementar essa atividade, como integrante da equipe de saúde. Nesse sentido, faz-se mister repensar os limites e a abrangência da pretensa autonomia do enfermeiro para prescrição de medicamentos, considerando-se o fato de que essa pode ser uma ação compartilhada entre médicos, enfermeiros, além de outros profissionais de nível universitário da área da saúde, desde que preencham os requisitos legais e detenham capacitação nesse âmbito. Portanto, a autonomia do profissional enfermeiro tende a se configurar a partir da identidade profissional, apresentando tensões com os aspectos sociais, políticos, mercadológicos e culturais nos quais os profissionais se inserem ${ }^{(10)}$.

\section{CONSIDERAÇÕES FINAIS}

Não basta a existência da Lei do Exercício Profissional da Enfermagem n. ${ }^{0} 7.498 / 86$ e do seu Decreto regulamentador $n .{ }^{\circ} 94.406 / 87$, para que seja assegurado o direito do enfermeiro prescrever medicamentos. Embora essa legislação contemple as atividades privativas do enfermeiro, é imprescindivel que a essa mesma legislação contemple os limites e a abrangência da atuação do enfermeiro, particularmente no que tange à prescrição de medicamentos por enfermeiros, bem como a solicitação de exames de rotina e complementares.

O enfermeiro, como profissional integrante da equipe de saúde, possui respaldo ético-legal para prescrever determinados medicamentos, porém dentro dos limites que a própria Lei do Exercício Profissional de Enfermagem (Lei n. ${ }^{0} 7.498 / 1986$ ) impõe, bem como as normatizações do Ministério da Saúde e as resoluções do COFEN que orientam em relação a essa atividade. Sendo assim, além do respaldo legal para prescrever medicamentos em determinadas circunstâncias, é imprescindivel que haja um investimento das instituições formadoras, das entidades representativas de classe, dos estabelecimentos de saúde e do próprio enfermeiro, no sentido de buscar uma capacitação profissional e uma constante atualização, que tornem possivel o exercício dessas atividades específicas. Assim, os currículos dos cursos de graduação de enfermagem devem, além de outras competências, capacitar 0 acadêmico para esta realidade, já que é rotina na atualidade a prática da prescrição medicamentosa por enfermeiros no mercado de trabalho. A fim de assegurar o exercício seguro e com isenção de riscos à clientela assistida, o enfermeiro poderá solucionar os problemas de saúde detectados, integrando às ações de enfermagem às ações multiprofissionais. Desse modo, a prescrição de medicamentos por enfermeiros não pode ser vista como uma atividade isolada, mas algo complementar à consulta de enfermagem, com os objetivos de conhecer e intervir sobre os problemas de saúde/doença, englobando outras ações, tais como a solicitação de exames de rotina e complementares. Convém ressaltar, também, que o enfermeiro, quando no exercício dessas atividades, responde integralmente pelos atos praticados, inclusive quando desses atos advirem situações de exposição dos clientes a riscos ou danos.

\section{REFERÊNCIAS}

1. Buchan J, Calman L. Implementing nurse prescribing: updated review of current practice internationally. Geneva (SWT): ICN; 2004.

2. Brasil. Lei n. ${ }^{\circ} 7.498$, de 25 de Junho de 1986 . Dispõe sobre a regulamentação do exercício da enfermagem e dá outras providências. In: Conselho Regional de Enfermagem do Estado de São Paulo. Documentos básicos de enfermagem: enfermeiros, técnicos e auxiliares. São Paulo (SP): COREN-SP; 2001.

3. Conselho Federal de Enfermagem (BR). Resolução 271/2002. Regulamenta as ações do enfermeiro na consulta, prescrição de medicamentos e requisição de exames. Rio de Janeiro (RJ): COFEN; 2002.

4. Conselho Federal de Enfermagem (BR). Resolução 195/1997. Dispõe sobre a solicitação de exames de rotina e complementares por enfermeiro. In: Conselho Regional de Enfermagem do Estado de São Paulo. Documentos básicos de enfermagem: enfermeiros, técnicos e auxiliares. São Paulo (SP): COREN-SP; 2001.

5. Brasil. Decreto-Lei n. ${ }^{\circ} 94.406$, de 08 de Junho de 1987. Regulamenta a Lei n. ${ }^{\circ}$ 7.498, de 25 de Junho de 1986, que dispõe sobre o exercício da enfermagem. In: Conselho Regional de Enfermagem do Estado de São Paulo. Documentos básicos de enfermagem: enfermeiros, técnicos e auxiliares. São Paulo (SP): COREN-SP; 2001.

6. Estado de São Paulo. Lei n. 10.241 , de 18 de março de 1999. Dispõe dos direitos dos usuários dos serviços e ações de saúde. Diário Oficial do Estado de São Paulo, 18 março 1999. Seção 51, p.1.

7. Cassiani SHDB, Coimbra JAH. Responsabilidade da enfermagem na administração de medicamentos: algumas reflexões para uma prática segura com qualidade de assistência. Rev Latino-am Enfermagem 2001;9(2):56-60.

8. United Kingdom Central Council for Nursing (UK). Midwifery and health visiting. Standards for the administration of medicines. London (UK): UKCC; 1992.

9. Santos EF. As instituições formadoras e a identidade profissional da enfermagem: mimetismo ou metamorfose (dissertação). Rio de Janeiro (RJ): Universidade do Estado do Rio de Janeiro; 2005.

10. Gomes AMT. A autonomia profissional de enfermagem em saúde pública: um estudo de representações sociais (dissertação). Rio de Janeiro (RJ): Universidade do Estado do Rio de Janeiro; 2002. 